\title{
Detection of Choroidal Neovascularisation in Flat Irregular Pigment Epithelial Detachment in Central Serous Chorioretinopathy Using Optical Coherence Tomography Angiography: en-face Image Combined With Cross-Sectional Image
}

\section{So Jung Ryu}

Hanyang University Seoul Hospital https://orcid.org/0000-0002-9648-8500

\section{Jong Sub Lee}

Hanyang University Seoul Hospital

\section{Sang Hyup Lee}

Hanyang University Seoul Hospital

\section{Seong Joon Ahn}

Hanyang University Seoul Hospital

Byung Ro Lee ( $\sim$ brlee@hanyang.ac.kr)

Hanyang University College of Medicine

\section{Research article}

Keywords: Choroidal neovascularisation, Flat irregular pigment epithelial detachment, Central serous chorioretinopathy, Optical coherence tomography angiography, En-face image, Cross-sectional image

Posted Date: August 14th, 2020

DOl: https://doi.org/10.21203/rs.3.rs-57417/v1

License: (c) (i) This work is licensed under a Creative Commons Attribution 4.0 International License. Read Full License 


\section{Abstract}

Background: Central serous chorioretinopathy (CSC) is a disorder characterised by serous detachment of the retina and is associated with retinal pigment epithelium (RPE) alteration. Flat irregular pigment epithelium detachment (FIPED) is one of the pigment epithelial detachment (PED) patterns of CSC and it is associated with chronic CSC.

This study investigated choroidal neovascularisation (CNV) in FIPED with en-face optical coherence topography angiography (OCTA) and cross-sectional OCTA, to evaluate the incidence of CNV and compare the efficacy of each method.

Methods: We retrospectively studied OCT and OCTA images of 328 eyes with CSC. OCTA B-scans and macular cube were primarily reviewed for the detection of FIPED and CNV. En-face OCTA and crosssectional OCTA with Angio-B view, which is an image that combines an OCT B-scan with a flow signal were analysed to evaluate the presence of CNV in FIPED.

Results: CNV was observed in 23 eyes on en-face OCTA and 21 eyes on cross-sectional OCTA, among 93 eyes of 88 patients with FIPED. There were eight discrepant cases, in which result of en-face OCTA was not equal to that of cross-sectional OCTA.

Conclusions: The CNV and FIPED lesions were well detected on cross-sectional and en-face OCTA. Integration of these two methods can potentially improve the utility and diagnostic accuracy of OCTA.

\section{Background}

Central serous chorioretinopathy (CSC) is a disorder characterised by serous detachment of the neurosensory retina and is associated with retinal pigment epithelium (RPE) alteration. It is predominantly encountered in young or middle-aged individuals $[1,2]$.

In fluorescein angiography (FA), diffuse RPE defects can be visualised and a single point or multifocal leakage points are observed according to the degree of chronicity. Indocyanine green angiography (ICGA) shows dilation of large choroidal veins in the mid-phase. Choroidal vascular hyperpermeability and congestion are thought to be the main causes of CSC development [3]. Optical coherence tomography (OCT) can identify various aspects of RPE alterations, according to the CSC chronicity [4].

Flat irregular pigment epithelium detachment (FIPED) is one of the pigment epithelial detachment (PED) patterns of CSC. It is mainly observed in chronic CSC compared with acute CSC. It has an irregular RPE elevation compared to a dome-shaped PED. Most FIPEDs are known to be avascular, however, choroidal neovascularisation (CNV) in FIPED has been reported in long-standing CSC cases [5, 6]. Because of multiple RPE changes and dilated pachy-vessels of the choroid, it is often difficult to identify CNV on FA or ICGA [7]. 
Optical coherence tomography angiography (OCTA) is a new imaging modality that can detect blood flow without invasive dye injection. It can effectively identify the presence of CNV in FIPED as an en-face image $[8,9]$. Swept-source OCTA can detect blood flow under the RPE and can be identified by layer, which is advantageous over FA and ICGA.

However, the analysis using an en-face image in the existing OCTA protocol may result in errors, such as motion artefacts, projection artefacts, layer segmentation errors, and flow signal masking that can occur during the calibration of projection artefacts. En-face imaging is challenging because the size of FIPED is small to be accurately divided and analysed into layers. Manually correcting the layer segmentation for each cross-sectional image may be feasible only for research purposes; however, it is impractical to rely on automated computer analysis.

To overcome the limitations of this clinical application, the hardware and software of OCTA was upgraded, and cross-sectional OCTA was introduced. Cross-sectional OCTA provides an image obtained by adding the flow signal to the existing ОСТ B-scan with false code. It is possible to observe the flow signal in the PED without introducing errors in the process of creating the en-face image.

This study investigated CNV in FIPED by two methods, en-face OCTA and cross-sectional OCTA, to evaluate the incidence of CNV and compare the efficacy of both.

\section{Methods}

\section{Study design and ethical statement}

We retrospectively reviewed the medical records of 290 patients (328 eyes) with CSC who visited the Department of Ophthalmology at Hanyang University Hospital between February 1, 2016, and February 1, 2018. This study was conducted according to the tenets of the Declaration of Helsinki and was approved by the Institutional Review Board at Hanyang University Seoul Hospital.

Eyes with other retinal abnormalities, such as polypoidal choroidal vasculopathy, age-related macular degeneration, idiopathic choroidal neovascularisation or other retinal vascular diseases, intraocular inflammation, and posterior segmental tumour were excluded. In addition, we excluded patients who had steroid-induced CSC and organ transplant-associated CSC. According to the manufacturer's recommendation [10], images of poor quality (image quality score lower than 45 ) provided by the onboard OCT software were excluded.

\section{Definitions}

The definitions used in this study were adapted from previous reports [2]. CSC was defined as a retinal disease characterised by serous detachment of the neurosensory retina secondary to one or more focal lesions of RPE [11]. Chronic CSC was defined as presence of symptoms for more than six months, history of recurrence, background fundus change, such as atrophic retinal and RPE changes, visible drainage tract or other atypical dye leakages during FA initial examination, and chronic CSC $[4,12]$. Intravitreal anti- 
Vascular endothelial growth factor (VEGF) injection and photodynamic therapy (PDT) history were not excluded from the study criteria.

FIPED was defined as a small, flat, and irregular surface PED on the OCT B-scan. Unlike semi-circular PED, RPE elevation is not large but the RPE layer is separated from Bruch's membrane and a "double layer sign" is observed [13]. The sub-RPE space at the site of FIPED showed either hypo-reflection (not optically filled) or at least partial hyper-reflection, as previously described [7].

\section{Study protocol}

Patients' medical records were reviewed, including best-corrected visual acuity, spherical equivalent, dilated fundus biomicroscopy findings, and multimodal imaging data including OCT, FA, ICGA, and OCTA.

All subjects underwent OCT and OCTA with Swept-source optical coherence tomography (SS-OCT) (Deep range imaging OCT, Triton, Topcon, Tokyo, Japan). We identified the presence of FIPED on OCT B-scans (Topcon SS-OCT parameters: $100 \mathrm{KHz}$, A-scan rate, wavelength $1050 \mathrm{~nm}$ ). This study used OCTA ratio analyses employed by Topcon, which is an intensity ratio analysis, not based on amplitude-decorrelation. A $3 \times 3 \mathrm{~mm}$ volume scan was performed, and each B-scan's position was automatically scanned four times. All OCT and OCTA assessments were performed by two trained retinal specialists (B.R.L. and S.J.A.) who were not aware of each other's imaging findings. OCTA B-scans and the $3 \times 3 \mathrm{~mm}$ OCTA macular cube were primarily reviewed for the detection of FIPED and the presence or absence of CNV at the site of the FIPED. If a $3 \times 3 \mathrm{~mm}$ OCTA image did not show any FIPED, the findings were confirmed through evaluation of the $4.5 \times 4.5 \mathrm{~mm}$ or $6 \times 6 \mathrm{~mm}$ OCTA B-scans.

\section{Choroidal neovascularisation using en-face OCTA and cross-sectional OCTA}

When FIPED was confirmed, the OCTA image was analysed to evaluate the presence of CNV in FIPED. Using OCTA software, the retinal layers and generated en-face images of the superficial plexus, deep plexus, avascular retina, and choriocapillaris were automatically segmented. The inner plexiform layer $(\mathrm{IPL}) /$ inner nuclear layer (INL) line and the basement membrane (BM) line were created by automated segmentation. Next, the en-face image of the outer retina (from $70.2 \mu \mathrm{m}$ under IPL/INL line to BM line) and choriocapillaris (from BM line to $10.4 \mu \mathrm{m}$ under BM line) was constructed. For en-face OCTA, these two images were analysed to evaluate the presence of CNV in FIPED.

The OCT B-scans were then combined with the corresponding en-face OCTA images, and scrolling was enabled through the sections similar to that of OCT cube scan. In the cross-sectional OCTA, we used the Angio-B view provided by the Topcon, which is an image that combines an OCT B-scan with a flow signal coated with a false-colour code. The cross-sectional view helped identify the flow signal in the FIPED by providing scrolling function. It was possible to distinguish between projection artefacts and the real flow signals by checking the sequential sections.

\section{Results}




\section{Study population}

This study retrospectively analysed the medical records of 290 patients (328 eyes), of which 256 patients (279 eyes) were actually included in the final analysis. In the remaining 15 patients (26 eyes), OCTA was not performed or the image quality was found to be poor. Age-related macular degeneration (AMD) was suspected on FA and ICGA in seven patients (10 eyes). A total of 13 eyes (12 patients) with other retinal diseases were excluded. FIPED was detected in 93 eyes of 88 patients (approximately 33.3\%). The mean age of patients was $55.34 \pm 11.86$ years, and $79.6 \%$ (74 eyes, 69 patients) had chronic CSC.

\section{Choroidal neovascularisation in FIPED}

CNV was observed in 23 eyes on en-face OCTA and 21 eyes on cross-sectional OCTA, among 93 eyes with FIPED. Actual CNV was noted in 21 eyes. The specificity and sensitivity of CNV detection using enface OCTA alone and in combination with cross-sectional OCTA are shown in Table 1. There was no significant difference in terms of in age $(p>0.05 ; 59.41 \pm 9.49$ years for those with CNV, $51.29 \pm 11.93$ years for those without CNV). All 21 cases of CNV were detected in those with chronic CSC.

Table 1. Sensitivity and specificity of detection of choroidal neovascularisation

\begin{tabular}{|ll|}
\hline Imaging modality & Sensitivity/specificity \\
\hline En-face OCTA alone & $85.7 \%(18 / 21)$ \\
\cline { 2 - 2 } & $93.1 \%(67 / 72)$ \\
\hline En-face OCTA with cross-sectional OCTA & $100 \%(21 / 21)$ \\
\cline { 2 - 2 } & $100 \%(72 / 72)$ \\
\hline
\end{tabular}

OCTA - Optical coherence tomography angiography

\section{Case descriptions}

CNV in FIPED was observed in 21 eyes with cross-sectional OCTA and 23 eyes with en-face OCTA. There were eight discrepant cases, in which CNV was observed on en-face OCTA, but not on cross-sectional OCTA. In the remaining cases, however, cross-sectional OCTA, but not en-face OCTA, could detect CNV. Figure 1-3 introduce several cases of correspondence and discrepancy.

\section{No discrepancy in en-face OCTA and cross-sectional OCTA}

Figure 1 shows OCTA images of four cases among 18 eyes in which there was no discrepancy in the results of en-face and cross-sectional OCTA. Figure 1-(A), (B), (C), and (D) are the $3 \times 3 \mathrm{~mm}$ sections of enface OCTA and Figure 1-(E), $(F),(G)$, and $(H)$ are the corresponding cross-sectional OCTA images. Both enface OCTA and cross-sectional OCTA could detect CNV in FIPED. Figure 2 and 3 show cases of discrepancy between the two methods. 


\section{Discrepancy in en-face OCTA and cross-sectional OCTA}

Figure 2 shows two cases of CNV in the en-face view [in (A) and (B)], however, absence of CNV was confirmed by cross-sectional OCTA [in (C) and (D)] among a total of 5 cases (5.38\%). Among them, 4 cases were identified as projection artefacts and 1 due to a segmentation error because of the choriocapillaris layer appearing under the FIPED. Figure 3 shows discrepancy of the CNV on en-face OCTA in 3 eyes (3.23\%). A clear flow signal was observed in the cross-sectional OCTA; however, PED size was small, and it was masked in the process of removing projection artefacts in the en-face OCTA. Thus, among the 93 eyes, 21 cases of CNV in FIPED were identified and 8 cases showed discrepant results.

\section{Discussion}

In this study, of the 93 eyes with FIPED, CNV was observed in 21 eyes. The prevalence of CNV was 22.6\%, which was closer to the lower border reported in previous studies. Previous reports have confirmed the presence of CNV in $18.9-58 \%$ of chronic CSC cases [7, 8, 13].

Using OCTA, several studies have quantified vessel density and flow index [14], as well as CNV $[15,16]$. Accurate segmentation is important to interpret and quantify OCTA findings. However, in eyes with retinal pathology such as chronic CSC, the margins of the retinal layer are distorted and precise boundary segmentation can be difficult. Although many researchers have attempted to improve the quality of automated segmentation in diseased eyes $[17,18]$, accurate automated segmentation is not achieved in all clinical cases. Therefore, manual correction of segmentation is often required [19, 20]. In cases of chronic CSC, most FIPEDs are known to be avascular, although CNV in FIPED has been reported $[5,6]$. However, because of multiple RPE changes and dilated pachy-vessels of the choroid, it is often difficult to detect CNV on FAG or ICGA [7].

Recently, several studies on PED using en-face views in OCTA have been performed $[8,9,13,21]$. However, analysis using en-face images may result in errors, such as motion artefacts, projection artefacts, layer segmentation errors, and flow signal masking that can occur during the calibration of projection artefacts. To complement this, cross-sectional OCTA obtained by adding the flow signal to the existing OCT B-scan with false code, was introduced. It is possible to observe the flow signal in the PED without being affected by the errors that may occur in creating the en-face image. This results in continuous sectional views on scrolling. In cases of PCV and PED in AMD, some studies using cross-sectional OCTA as well as en-face view have been published [22-25].

It is important that the entire morphology of a CNV lesion is thoroughly analysed to qualitatively define a choroidal neovascular network, based on the shape, branching, anastomoses, type of vessels termini, and presence of hypointense perilesional halo [26]. However, there could be some errors in en-face OCTA due to the abovementioned difficulties. Since CNV is not a coplanar structure, it may show a different morphology at each level of depth. En-face OCTA provides depth-resolved images; therefore, manual confirmation of the cross-sectional B-scan allows a layer-by-layer tomographic visualisation of the entire neovascular feature. 
This study is significant because of the identification of CNV on FIPED of chronic CSC using both en-face and cross-sectional OCTA. The CNV in FIPED was observed in 23 eyes on en-face OCTA and 21 eyes on cross-sectional OCTA. The diagnostic efficacy of both the methods was similar; however, eight cases showed discrepant results on en-face OCTA. Among them, five were false-positive cases, i.e., en-face OCTA detected the presence of CNV but there was no actual CNV in these eyes (Figure 2). Four cases were identified as projection artefacts and one case as a segmentation error with the choriocapillaris layer under FIPED. The remaining 3 eyes had actual CNV confirmed on cross-sectional OCTA, but no CNV was seen on en-face OCTA. The reason for the false negativity was the small size of PED that could not be detected on en-face OCTA and masked PED in the process of removing projection artefacts. A total of eight eyes showed discrepant results on en-face OCTA that could not be ignored, and cross-sectional OCTA was required to compensate for these errors. With cross-sectional OCTA, a more complete layer-bylayer analysis of the entire structure could be performed to detect the precise location of the CNV lesion relative to the RPE layer.

When FIPED is observed in chronic CSC, it is not necessary to consider anti-VEGF treatment for the possibility of type $1 \mathrm{CNV}$. In this study, CNV in FIPED was found in 21 out of 93 eyes, and less than onethird of cases of active CNV. Previous studies have reported that OCTA findings in PED were caused by the severe choriocapillaris alteration in those with $\operatorname{AMD}[16,27]$. However, the vascular signal in FIPED with chronic CSC seems to be due to compensatory choriocapillaris vascular remodelling owing to a weak Bruch's membrane. The CNV lesion described as a "darker halo" or total absence of choriocapillaris with loss of both the inner and deeper choroidal vessels encircling the CNV lesions could not be found in this study. Therefore, careful observation in these cases compared to AMD is recommended.

Limitations of this study are the retrospective design and relatively small sample size. Additionally, this study investigated the strength of cross-sectional views, and unlike for the en-face view, clinicians need to observe each CNV lesion using manual scrolling. In addition, longitudinal studies are needed to confirm changes in CNV lesions in FIPED in chronic CSC patients. However, this study has value in that it is the first study to analyse CNV in FIPED in chronic CSC patients using both en-face and cross-sectional OCTA.

\section{Conclusion}

In conclusion, this study demonstrates the potential feasibility of using cross-sectional OCTA to detect CNV. To identify CNV in FIPED without dye angiography, the reliability of detecting flow signal within any pathological lesion is critical. The clinical use of cross-sectional OCTA, as well as en-face OCTA to interpret FIPEDs may help clinicians in decision-making. Integration of these two methods can potentially improve the utility and diagnostic accuracy of OCTA.

\section{Abbreviations}

CSC: Central serous chorioretinopathy; RPE: Retinal pigment epithelium; FA: Fluorescein angiography; ICGA: Indocyanine green angiography; OCT: Optical coherence tomography; FIPED; Flat irregular pigment 
epithelium detachment; PED: Pigment epithelial detachment; CNV: Choroidal neovascularisation; OCTA: Optical coherence tomography angiography; VEGF: Vascular endothelial growth factor; PDT:

Photodynamic therapy; SS-OCT: Swept-source optical coherence tomography; IPL: Inner plexiform layer; INL: Inner nuclear layer; BM: Basement membrane; AMD: Age-related macular degeneration

\section{Declarations}

\section{Acknowlegments}

Not applicable.

\section{Authors' contributions}

SJR and JSL contributed equally to this work.

Conception and design: SJR, SHL, SJA, and BRL; Data collection: SJR, SHL, SJA, and BRL; Analysis and interpretation: SJR, SHL, SJA, and BRL; Writing the article SJR, JSL, SHL, SJA, and BRL, Critical revision of the article; SJR, JSL, SHL, SJA, and BRL, Final approval of the article; SJR, SHL, SJA, BRL. All authors have read and approved the manuscript.

\section{Funding}

This work was supported by the research fund of Hanyang University(HY-2020)

\section{Availability of data and materials}

The datasets used and/or analysed during the current study are available from the corresponding author on reasonable request.

\section{Ethics approval and consent to participate}

This study adhered to the tenets of the Declaration of Helsinki. This study was approved by our Institutional Review Board.

\section{Consent for publication}

Not applicable.

\section{Competing interests}

The authors declare that they have no competing interests.

\section{Author details}

${ }^{1}$ Department of Ophthalmology, Hanyang University Hospital, Hanyang 
University College of Medicine, 222-1, Wangsimni-ro, Seongdong-gu, Seoul

04763, South Korea.

${ }^{2}$ Department of Ophthalmology, Hanyang University

Seoul Hospital, Seoul, South Korea.

Financial Support: This work was supported by the research fund of Hanyang University (HY-2020).

Conflicts of Interest: None of authors has any financial/conflicting interests to disclose.

\section{References}

1. Lane C: Stereoscopic Atlas of Macular Diseases: Diagnosis and Treatment. Br J Ophthalmo/ 1988, 72(9):720-720.

2. Spaide RF, Campeas L, Haas A, Yannuzzi LA, Fisher YL, Guyer DR, Slakter JS, Sorenson JA, Orlock DA: Central serous chorioretinopathy in younger and older adults. Ophthalmology 1996, 103(12):2070-2079; discussion 2079-2080.

3. Yannuzzi LA, Shakin JL, Fisher YL, Altomonte MA: Peripheral retinal detachments and retinal pigment epithelial atrophic tracts secondary to central serous pigment epitheliopathy.

Ophthalmology 1984, 91(12):1554-1572.

4. Song IS, Shin YU, Lee BR: Time-periodic characteristics in the morphology of idiopathic central serous chorioretinopathy evaluated by volume scan using spectral-domain optical coherence tomography. Am J Ophthalmol 2012, 154(2):366-375 e364.

5. Fung AT, Yannuzzi LA, Freund KB: Type 1 (sub-retinal pigment epithelial) neovascularization in central serous chorioretinopathy masquerading as neovascular age-related macular degeneration. Retina 2012, 32(9):1829-1837.

6. Daruich A, Matet A, Dirani A, Bousquet E, Zhao M, Farman N, Jaisser F, Behar-Cohen F: Central serous chorioretinopathy: Recent findings and new physiopathology hypothesis. Prog Retin Eye Res 2015, 48:82-118.

7. Hage R, Mrejen S, Krivosic V, Quentel G, Tadayoni R, Gaudric A: Flat irregular retinal pigment epithelium detachments in chronic central serous chorioretinopathy and choroidal neovascularization. Am J Ophthalmol 2015, 159(5):890-903 e893.

8. Quaranta-El Maftouhi M, El Maftouhi A, Eandi CM: Chronic central serous chorioretinopathy imaged by optical coherence tomographic angiography. Am J Ophthalmol 2015, 160(3):581-587 e581.

9. Bonini Filho MA, de Carlo TE, Ferrara D, Adhi M, Baumal CR, Witkin AJ, Reichel E, Duker JS, Waheed NK: Association of Choroidal Neovascularization and Central Serous Chorioretinopathy With Optical Coherence Tomography Angiography. JAMA Ophthalmol 2015, 133(8):899-906. 
10. Mansouri K, Medeiros FA, Tatham AJ, Marchase N, Weinreb RN: Evaluation of retinal and choroidal thickness by swept-source optical coherence tomography: repeatability and assessment of artifacts. Am J Ophthalmol 2014, 157(5):1022-1032.

11. Wang M, Munch IC, Hasler PW, Prunte C, Larsen M: Central serous chorioretinopathy. Acta Ophthalmol 2008, 86(2):126-145.

12. Ross A, Ross AH, Mohamed Q: Review and update of central serous chorioretinopathy. Curr Opin Ophthalmol 2011, 22(3):166-173.

13. Bousquet E, Bonnin S, Mrejen S, Krivosic V, Tadayoni R, Gaudric A: Optical Coherence Tomography Angiography of Flat Irregular Pigment Epithelium Detachment in Chronic Central Serous Chorioretinopathy. Retina 2018, 38(3):629-638.

14. Ishibazawa A, Nagaoka T, Takahashi A, Omae T, Tani T, Sogawa K, Yokota H, Yoshida A: Optical Coherence Tomography Angiography in Diabetic Retinopathy: A Prospective Pilot Study. Am J Ophthalmol 2015, 160(1):35-44 e31.

15. Jia Y, Bailey ST, Hwang TS, McClintic SM, Gao SS, Pennesi ME, Flaxel CJ, Lauer AK, Wilson DJ, Hornegger $\mathrm{J}$ et al: Quantitative optical coherence tomography angiography of vascular abnormalities in the living human eye. Proc Natl Acad Sci U S A 2015, 112(18):E2395-2402.

16. Jia Y, Bailey ST, Wilson DJ, Tan O, Klein ML, Flaxel CJ, Potsaid B, Liu JJ, Lu CD, Kraus MF et al: Quantitative optical coherence tomography angiography of choroidal neovascularization in agerelated macular degeneration. Ophthalmology 2014, 121(7):1435-1444.

17. Chiu SJ, Izatt JA, O'Connell RV, Winter KP, Toth CA, Farsiu S: Validated automatic segmentation of AMD pathology including drusen and geographic atrophy in SD-OCT images. Invest Ophthalmol Vis Sci 2012, 53(1):53-61.

18. Srinivasan PP, Heflin SJ, Izatt JA, Arshavsky VY, Farsiu S: Automatic segmentation of up to ten layer boundaries in SD-OCT images of the mouse retina with and without missing layers due to pathology. Biomed Opt Express 2014, 5(2):348-365.

19. Teng P-y: Caserel - An Open Source Software for Computer-aided Segmentation of Retinal Layers in Optical Coherence Tomography Images; 2013.

20. Yin X, Chao JR, Wang RK: User-guided segmentation for volumetric retinal optical coherence tomography images. J Biomed Opt 2014, 19(8):086020.

21. Dansingani KK, Balaratnasingam C, Klufas MA, Sarraf D, Freund KB: Optical Coherence Tomography Angiography of Shallow Irregular Pigment Epithelial Detachments In Pachychoroid Spectrum Disease. Am J Ophthalmol 2015, 160(6):1243-1254 e1242.

22. Kang H, Byeon SH, Kim SS, Koh HJ, Lee SC, Kim M: Combining En Face Optical Coherence Tomography Angiography with Structural Optical Coherence Tomography and Blood Flow Analysis for Detecting Choroidal Neovascular Complexes in Pigment Epithelial Detachments. Retina 2019, 39(8):1551-1561.

23. Tan ACS, Freund KB, Balaratnasingam C, Simhaee D, Yannuzzi LA: Imaging of Pigment Epithelial Detachments with Optical Coherence Tomography Angiography. Retina 2018, 38(9):1759-1769. 
24. Cheung CMG, Yanagi Y, Akiba M, Tan A, Mathur R, Chan CM, Yeo I, Wong TY: Improved Detection and Diagnosis of Polypoidal Choroidal Vasculopathy Using a Combination of Optical Coherence Tomography and Optical Coherence Tomography Angiography. Retina 2019, 39(9):1655-1663.

25. Chan SY, Wang Q, Wang YX, Shi XH, Jonas JB, Wei WB: Polypoidal Choroidal Vasculopathy Upon Optical Coherence Tomographic Angiography. Retina 2018, 38(6):1187-1194.

26. Coscas GJ, Lupidi M, Coscas F, Cagini C, Souied EH: OPTICAL COHERENCE TOMOGRAPHY ANGIOGRAPHY VERSUS TRADITIONAL MULTIMODAL IMAGING IN ASSESSING THE ACTIVITY OF EXUDATIVE AGE-RELATED MACULAR DEGENERATION: A New Diagnostic Challenge. Retina 2015, 35(11):2219-2228.

27. Moult E, Choi W, Waheed NK, Adhi M, Lee B, Lu CD, Jayaraman V, Potsaid B, Rosenfeld PJ, Duker JS et al: Ultrahigh-speed swept-source OCT angiography in exudative AMD. Ophthalmic Surg Lasers Imaging Retina 2014, 45(6):496-505.

\section{Figures}

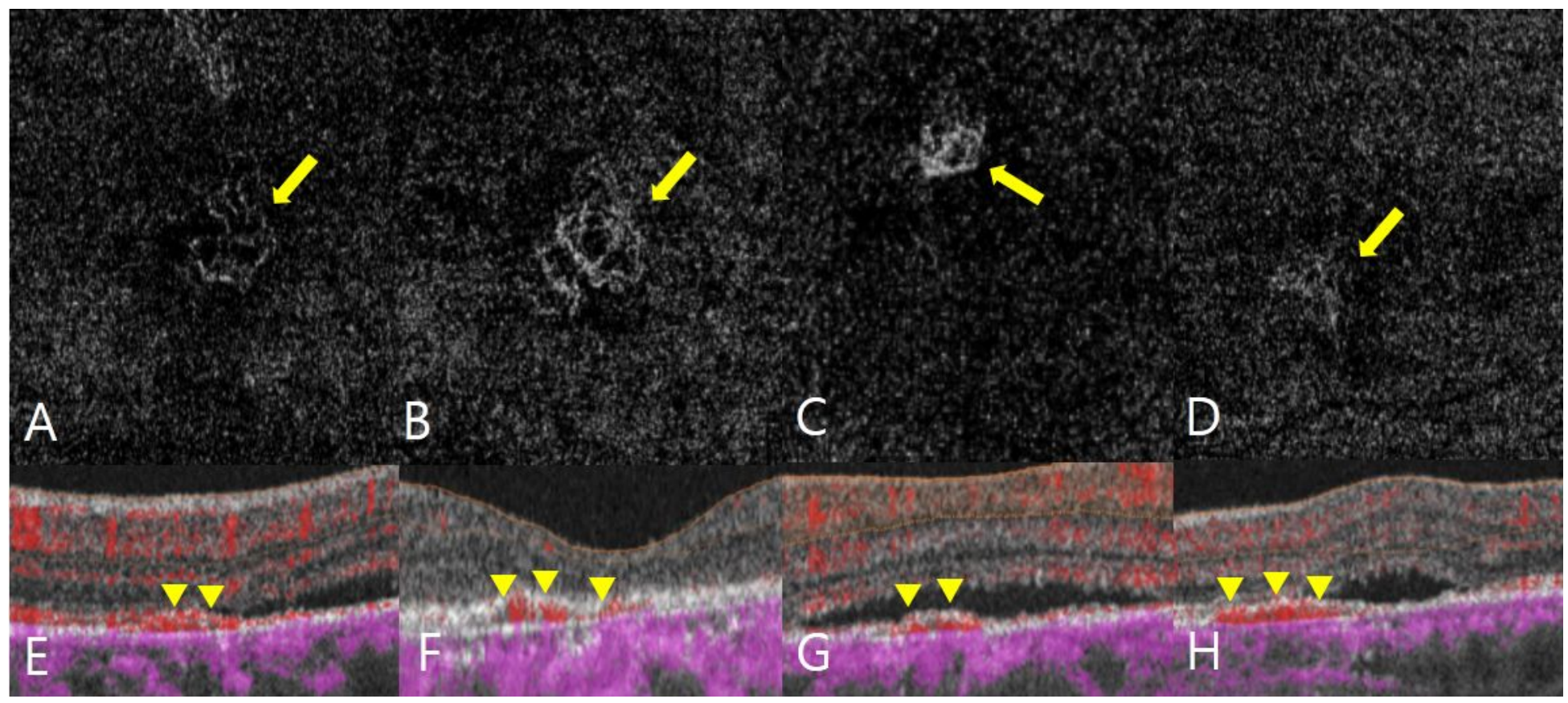

Figure 1

Representative cases of correspondence between the en-face view and the cross-sectional view. (A)-(D) The $3 \times 3 \mathrm{~mm}$ en-face view shows Choroidal neovascularisation (CNV) (arrows). (E)-(H) In the same lesions, cross-sectional views show the flow signal (arrow heads) in Flat irregular pigment epithelium detachment (FIPED). Both en-face view and cross-sectional view show CNV in FIPED. 


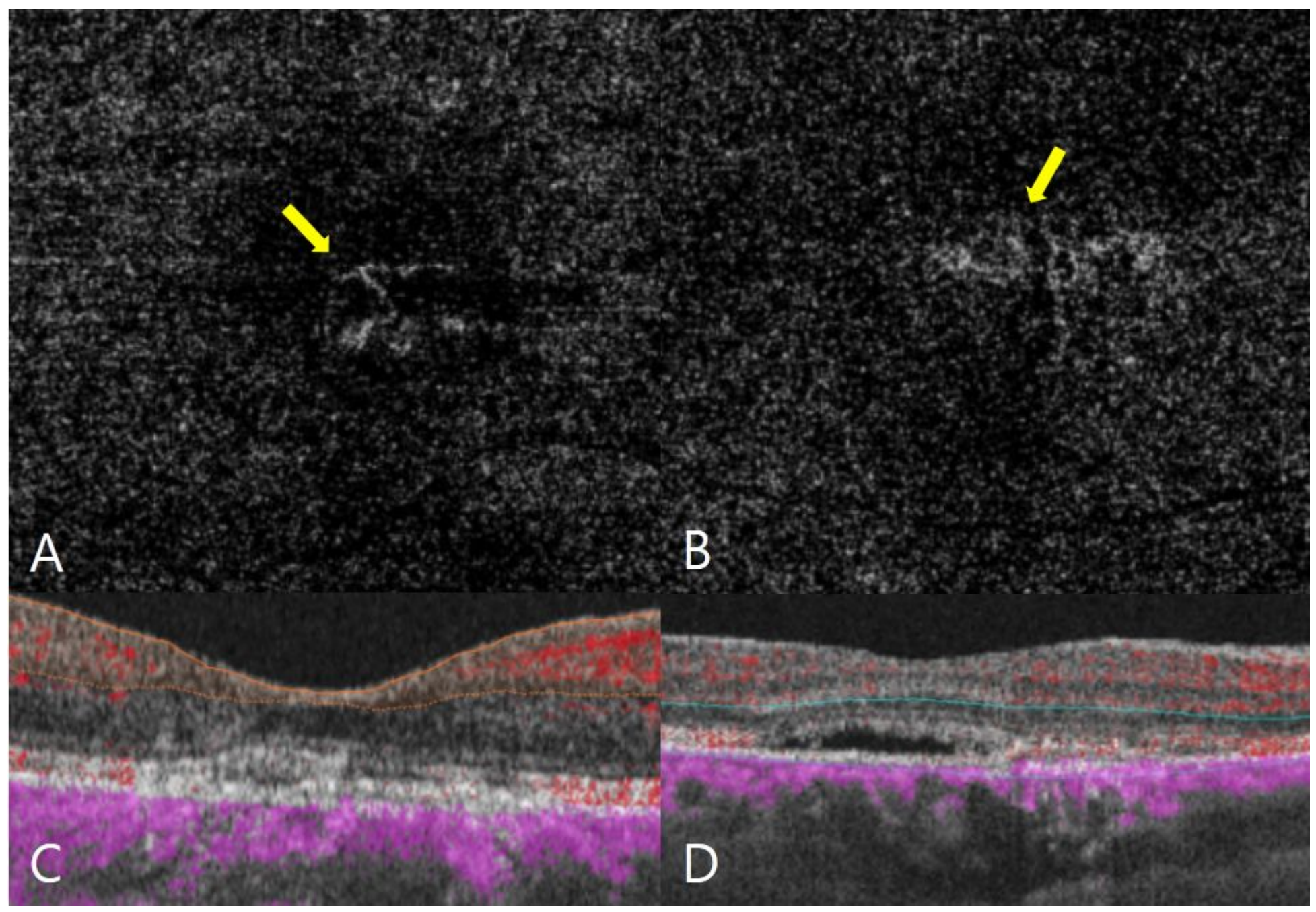

\section{Figure 2}

Representative cases of discrepancy between the en-face view and the cross-sectional view. Upper figures of the en-face view represent CNV (arrows) (A, B). There is no positive flow signal in the corresponding lesions in the cross-sectional views (C, D). There is no CNV in the pigment epithelial detachment when assessed by cross-sectional view in 5 cases $(5.38 \%)$. Among them, four cases were identified as projection artefacts and one case as a segmentation error due to the choriocapillary layer under FIPED. 


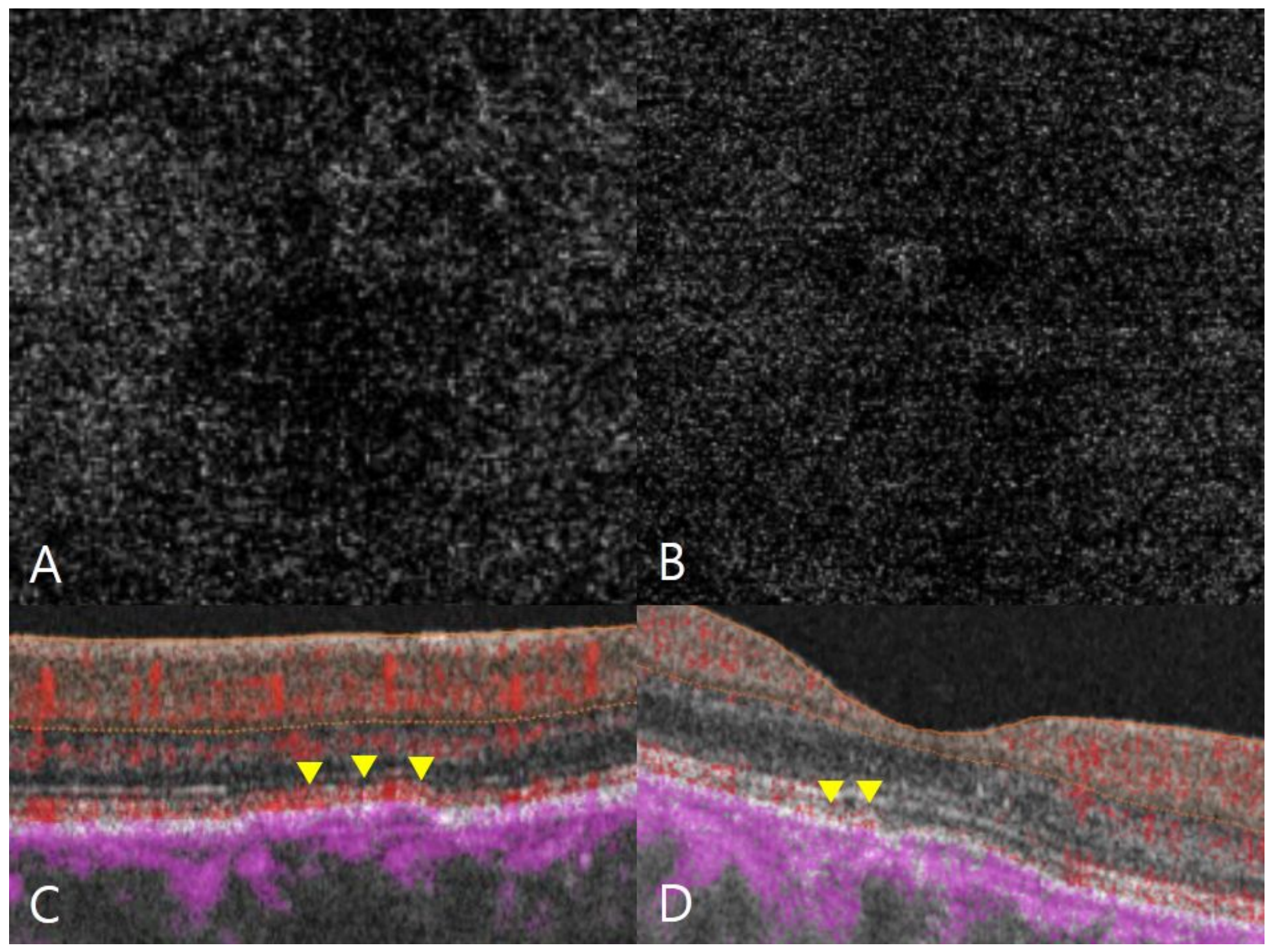

\section{Figure 3}

Representative cases of discrepancy contrary to Figure 2. The en-face views ( $A$ and $B$ ) do not show any $C N V$, but in $C$ and $D$, flow signals (arrowhead) can be seen in the cross-sectional view. A clear flow signal can be observed in the cross-sectiona I OCTA, but PED size appears very small, and it is masked in the process of removing projection artefacts in the en-face Optical coherence tomography angiography (OCTA). 